\title{
Traitement d'un sol effondrable par un liant hydrocarboné (bitume)
}

T. AYADAT

Maître de conférence à l'Universitê de M'Sila

M. DAHILI

K. MAIZ HADJ AHMED

Ingénieurs d'État en Génie civil
Le but de cette recherche expérimentale est l'amélioration des caractéristiques d'un sol dont le mouillage provoque son effondrement brusque. L'utilisation de tels terrains localisés dans la plupart des régions arides et semi-arides, nécessite obligatoirement de prendre des dispositions ou de traiter le sol, pour garantir une stabilité suffisante, et cela dépend généralement de la profondeur du sol, du type et de l'importance de l'ouvrage à implanter.

Après la réussite du traitement d'un sol effondrable par des liants hydrauliques (tels que le ciment C.PA 325 [1], la chaux [2] et le ciment alumineux fondu [3]), cette étude propose, d'une manière analogue, un traitement par un liant hydrocarboné (qui est le bitume) d'un sol effondrable de profondeur inférieure à $4 \mathrm{~m}$ et de déterminer ainsi les quantités d'eau, de bitume et l'énergie de compactage nécessaire à l'obtention d'un sol qu'on peut exploiter sans aucun problème de stabilité.

\section{Treatment of a collapsible soil with a hydrocarbon stabilising agent (bituminous binder)}

The objectif of this experimental work is to improve the properties of a subsidable soil known as a collapsing soil. The erection of structures on these type of solls. located in arid or semi-arid zones, necessarily calls for the treatment of these soils in order to either stabilize or cause collapse of the soll deposit prior to construction. The amount of treatment and type depend on the depth of the collapsible soil and the support requirement for the proposed facility.

After the effectiveness of treatment of collapsible soils by hydraulic stabilising agents (such as CPA 325 cement [1], lime [2] and similariy), this study examines the bituminous treatment of a such soil. at depth of less than $4 \mathrm{~m}$, and determines the minimum amounts of water, bituminous binder and compacting energy necessary for obtaining a no-collapsible soil. 


\section{Introduction}

L'existence des sols effondrables dans plusieurs régions du monde, ainsi que les problèmes qu'ils posent aux constructions, ont été reconnus depuis longtemps. Le manque d'informations sur leurs dépôts est dû principalement à leur localisation dans des régions arides, où le développement économique est très limité. Les progrès récents de l'irrigation et la modernisation des moyens de transport ont rendu possible l'exploitation de ces régions avec la construction de complexes industriels et urbanistiques. Les tassements importants qui résultent de l'utilisation de grandes quantités d'eau et les graves désordres subis par les ouvrages, ont donné dans ces trois dernières décennies, une impulsion à la recherche concernant ces sols, notamment les méthodes de leur identification et de leur traitement.

Dans plusieurs sites, il est possible de traiter le sol pour le stabiliser ou de provoquer son effondrement, avant d'y construire un ouvrage. La nature du traitement à appliquer dépend essentiellement de la profondeur du sol effondrable et du type de fondation.

Une grande variété de méthodes de traitement a été utilisée ou suggérée. Parmi elles, on peut citer:

- le traitement par compactage dynamique avec ou sans humidification du sol ;

- le traitement par pré-mouillage du sol ;

- le compactage par explosion ;

- le traitement par injection ;

- le traitement chimique, thermique ou aux ultrasons ; - le traitement par enfoncement de pieux ou de colonnes ballastées [4] :

- le compactage par vibrocompaction et vibroflottation.

Lorsque la couche de sol effondrable n'est pas trop épaisse, inférieure à $4 \mathrm{~m}$, il est souvent économique de la remplacer par un sol approprié [5], ou par le même sol excavé puis remis en place après traitement avec du ciment CPA 325 [1], de la chaux [2] ou du ciment alumineux fondu [3].

L'objectif de cette recherche est de montrer qu'il est aussi possible de traiter le sol effondrable excavé par du bitume avant sa remise en place.

\section{2}

\section{Matériaux, matériels et essais}

2.1

\section{Matériaux de l'étude}

Les essais ont été effectués sur un sol reconstitué composé de $80 \%$ de sable et de $20 \%$ de particules fines $(<80 \mu \mathrm{m})$, pour lequel l'application des différents critères d'effondrement, rapportés par Ayadat et al. [6], montrent que celui-ci est effondrable.

Le sable utilisé pour la reconstitution du sol effondrable est un sable de rivière extrait de la région de Bousaâda (Algérie). Il est très utilisé pour la confection du béton puisqu'il contient un faible pourcentage de particules fines. Pour éliminer les graviers et les cailloux, le sable est passé au tamis de $2 \mathrm{~mm}$, puis le tarnisat séché à l'étuve $\left(105^{\circ} \mathrm{C}\right)$ pendant 24 heures. Ses caractéristiques sont résumées comme suit :

- granulométrie comprise entre 0,1 et 2,0 mm, dont $3 \%$ des particules sont inférieures à $0,1 \mathrm{~mm}$;

- coefficient d'uniformité de 2,04 ;

- coefficient de courbure de 0,90;

- module de finesse de 1,41.

Le sol fin ( $D<80 \mu \mathrm{m})$ utilisé est extrait de Oultène de la région de Bousaâda, de couleur brune. Il possède les caractéristiques suivantes:

- limite de liquidité $\mathrm{w}_{\mathrm{L}}=43,9 \%$;

- limite de plasticité $w_{p}=20,4 \%$;

- densité spécifique $\mathrm{G}_{\mathrm{s}}=2,7$.

Le sol reconstitué est obtenu par le mélange de $80 \%$ de sable et de $20 \%$ de sol fin. Ses caractéristiques géotechniques sont données dans le tableau I et sa courbe granulométrique est représentée sur la figure 1.

Le bitume utilisé pour le traitement est une émulsion 60 utilisée comme couche d'accrochage dans les chaussées.

\begin{tabular}{|c|c|c|}
\hline TABLEAU I & \multicolumn{2}{|c|}{$\begin{array}{l}\text { Caractéristiques géotechniques du sol } \\
\text { reconstitué. }\end{array}$} \\
\hline \multicolumn{2}{|c|}{ Limite de liquicité $\left(\mathrm{W}_{1}\right)$} & $18,4 \%$ \\
\hline \multicolumn{2}{|c|}{ Limite de plasticité $\ll w_{p} \|$} & $9,3 \%$ \\
\hline \multicolumn{2}{|c|}{ Densité optimale u $\gamma_{\text {cot }}{ }^{2}$} & $2,06 \mathrm{~g} / \mathrm{cm}^{3}$ \\
\hline \multicolumn{2}{|c|}{ Teneur en eau optimale " w w } & $8,4 \%$ \\
\hline
\end{tabular}

Tamisats (\%)

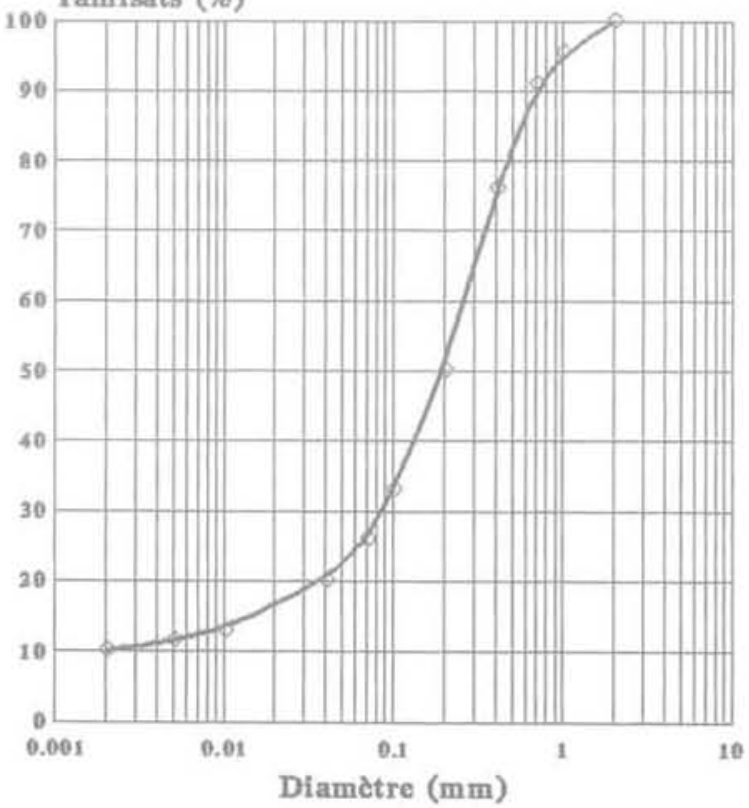

คG. 1 Analyse granulométrique du sol reconstitué. 


\section{Matériels d'essais}

\section{Dant}

\section{Appareillage spécifique}

- Moule cedométrique de 50,4 mm de diamètre et $20 \mathrm{~mm}$ de hauteur.

- Bâti de consolidation d'un bras de levier de 1/10.

- Jeu de poids.

\section{:ava}

\section{Appareil de compactage}

Il est composé :

- d'une tige verticale pour le guidage de $200 \mathrm{~mm}$ de hauteur et de 12,2 mm de diamètre. Elle est fixée à un disque horizontal de $5 \mathrm{~mm}$ d'épaisseur et de $50,2 \mathrm{~mm}$ de diamètre;

- d'un mouton (dame) de masse de $136 \mathrm{~g}$, en forme de disque ayant un trou centré de 12,25 mm, de $39,2 \mathrm{~mm}$ de diamètre et de 16,4 mm d'épaisseur (Fig. 2).

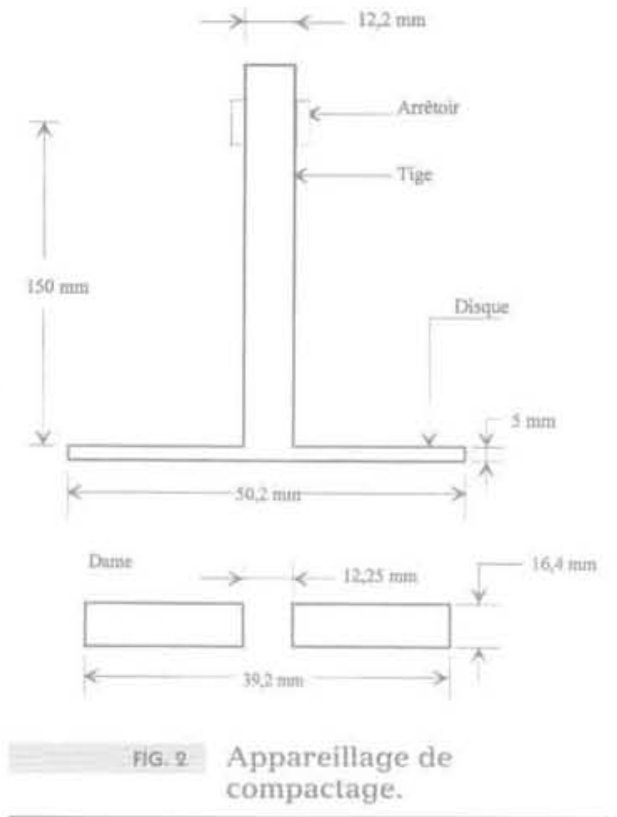

\section{3}

\section{Réalisation de l'essai œdométrique}

Le sol de l'essai est constitué d'un mélange homogène et sec de $20 \%$ du sol fin tamisé au tamis $0,08 \mathrm{~mm}$ et de $80 \%$ de sable passé au tanis $2 \mathrm{~mm}$. On ajoute une quantité d'eau distillée afin d'obtenir la teneur en eau désirée. L'ensemble sol et eau est malaxé dans un malaxeur électrique.

On chauffe alors la quantité voulue de bitume jusqu'à ce qu'il atteigne l'état liquide. On mélange rapidement par partie la quantité de sol reconstitué. Dès que l'ensemble (sol + bitume) devient homogène, on le place dans le moule œdométrique après un léger refroidissement. La gâchée de sol est versée dans la trousse cylindrique de l'œdomètre puis compactée en deux couches avec une certaine énergie de compactage (nombre de coups de mouton) donnée par le dispositif décrit ci-dessus. L'éprouvette est ensuite arasée au niveau du bord supérieur de la trousse ou de l'anneau avec grande précaution, à l'aide d'une lame rigide, pour obtenir une surface plane.

Le compactage consiste à laisser tomber la dame d'une hauteur $\mathrm{H}=150 \mathrm{~mm}$. Cette dame coulisse librement sur la tige verticale jusqu'à ce qu'elle percute le disque qui transmet le choc à l'échantillon (Fig. 3).

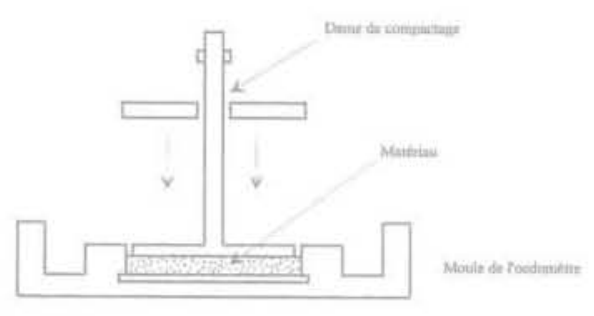

F6,3 Opération de compactage.

Après l'installation du moule cedométrique et mise au point, on effectue un essai de compressibilité conformément au mode opératoire du Laboratoire Central des Ponts et Chaussées à savoir, application successive toutes les 24 heures des charges suivantes : $25 ; 50 ; 100$ et $200 \mathrm{kPa}$ avec relevé des lectures du comparateur à $15 \mathrm{~s} ; 30 \mathrm{~s} ; 60 \mathrm{~s} ; 2$ min ; 5 min... et $24 \mathrm{~h}$. La particularité de cet essai proposé par Jennings et Knight [7], par rapport à l'essai normalisé de compressibilité, consiste à inonder l'échantillon après mesure du tassement sous $200 \mathrm{kPa}$ et à relever ensuite la nouvelle valeur après 24 heures de chargement de l'éprouvette saturée sous la même contrainte, puis à augmenter le chargement jusqu'à $400 \mathrm{kPa}$. On donne sur la figure 4, la courbe typique $e=f(\log \sigma)$ d'un tel sol, d'où on peut déduire le potentiel d'affaissement CP $(\%)$ par la relation :

$$
C P=\frac{\Delta e_{c}}{1+e_{0}} \times 100
$$

où : $\Delta e_{c}=e(200 \mathrm{kPa})-\mathrm{e}(200 \mathrm{kPa}$, inondé $)$

$\mathrm{e}_{0}=$ Indice des vides initial.

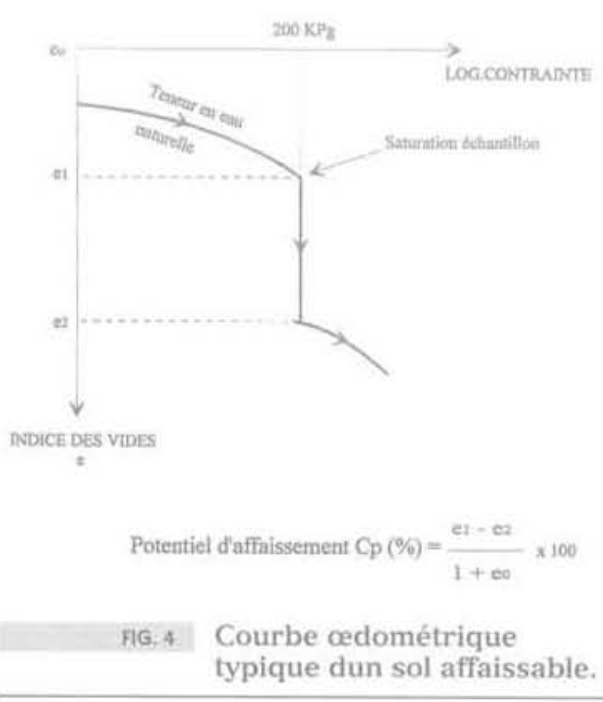


La valeur obtenue de CP est ensuite comparée aux valeurs de potentiel d'affaissement CP suggérées par Jennings et Knight [7] (Tableau II).

\begin{tabular}{c|c}
\hline \multicolumn{2}{c}{ TABLEAUII Valeurs du potentiel d'affaissement. } \\
\hline CP & Difficulté du problème \\
\hline $0 \%$ à $1 \%$ & Pas de problème \\
\hline $1 \%$ à $5 \%$ & Troubles modérés \\
\hline $5 \%$ a $10 \%$ & Troubles \\
\hline $10 \%$ à $20 \%$ & Troubles sévères \\
\hline$>20 \%$ & Troubles très sèvères \\
\hline
\end{tabular}

A la fin de l'essai, il est nécessaire de bien nettoyer l'anneau du reste des particules de bitume qui se sont collées sur les parois intérieures. A cet effet, il est nécessaire de prendre des précautions particulières de nettoyage pour éviter de rayer l'anneau et cela en plaçant tout l'appareillage dans un bac rempli de mazout avant tout nettoyage.

\section{Programme d'essais}

Les essais préliminaires du traitement du sol avec les pourcentages de bitume de 3 et $4 \%$ ont donné des résultats insuffisants. Pour cette raison les pourcentages ont été augmentés à $8 \%$ et plus.

De plus, les énergies de compactage, utilisées pour les essais du sol non traité, ont été elles aussi changées. En effet, les quelques essais effectués sur le sol traité avec les énergies de compactage de 10 à 25 coups ont donné des résultats insuffisants. Les nouveaux nombres de coups ont été 40,60 et 80 coups.

En se basant sur les résultats obtenus pour le sol non traité, montrant que l'effondrement augmente quand la teneur en eau diminue, on a décidé d'étudier le traitement du sol, avec seulement la teneur en eau minimale $(4 \%)$ qui représente l'état le plus défavorable.

Dans ces conditions, les valeurs retenues furent:

a) sol non traité

- teneurs en eau : $4 \%, 6 \%$ et $8 \%$,

- énergies de compactage: 10, 25 et 40 coups (Tableau III),

- teneur en bitume : $0 \%$;

b) sol traité

- teneurs en bitume : $8 \%, 10 \%, 12 \%, 14 \%$ et $16 \%$, - énergie de compactage: 40,60 et 80 coups (Tableau III),

-teneur en eau : $4 \%$.
TABLEAU ii Les différentes énergies de compactage.

\begin{tabular}{l|ccc}
\hline Degré de compactage & 1 & 2 & 3 \\
\hline Nombre de coups & 10 & 25 & $40^{*}$ \\
\hline & 40 & 60 & $80^{* *}$ \\
\hline Énergie Eu (kJoules) & 4,08 & 10,2 & $16,32^{*}$ \\
\hline & 16,32 & 24,48 & $32,64^{*+}$ \\
\hline
\end{tabular}

* Sol nan maité

" sol traile.

Eut=nimath jourics

Gid. Eu = Energie de compactape par unité de volume

in= Nombrote

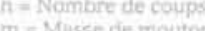

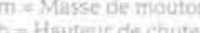

$g=$ Acceicerntion de la pesanteur

\section{4}

\section{Résultats et analyse}

\section{1}

\section{Sol non traité}

Les résultats, donnés dans le tableau IV et représentés sur les figures 5 et 6 , confirme le comportement observé pour les sols effondrables utilisés dans l'étude du traitement par du ciment CPA 325 [1], de la chaux [2] et du ciment alumineux fondu [3]. Ces résultats montrent que

Potentiel d'affaissement (CP \%)

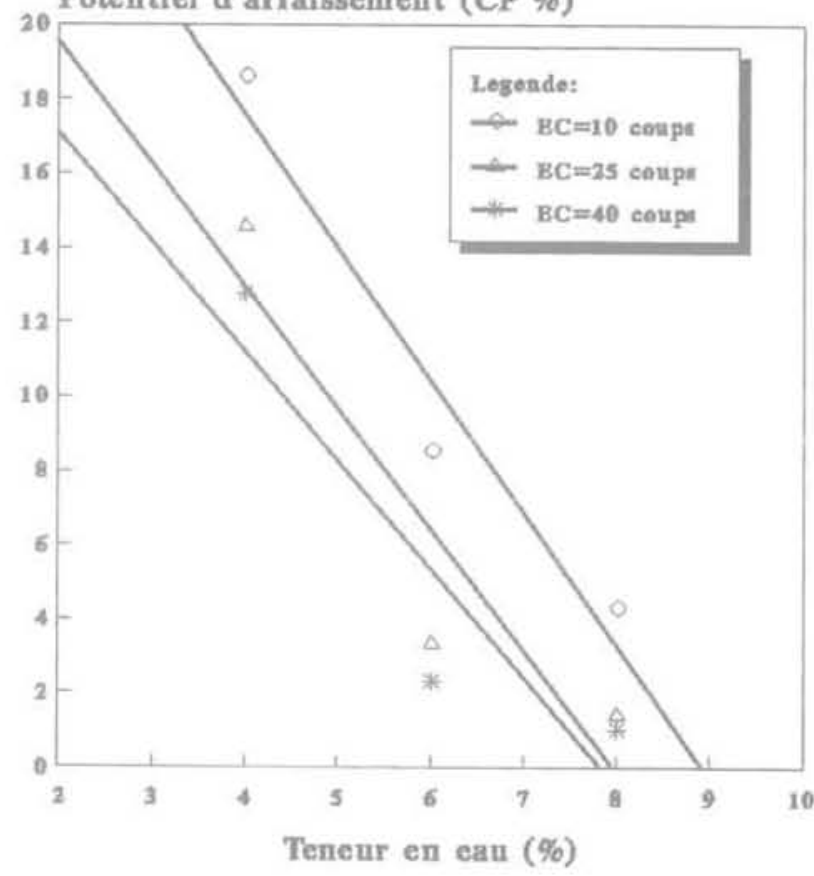

FIG.5 Influence de la teneur en eau sur CP (pour $0 \%$ de bitume).

TABLEAUIV Valeurs de CP pour sol non traité.

\begin{tabular}{|l|c|c|c|c|c|c|c|c|c}
\hline Nombre de coups & 10 & 25 & 40 & 10 & 25 & 40 & 10 & 25 & 40 \\
\hline W $(\%)$ & 4 & 4 & 4 & 6 & 6 & 6 & 8 & 8 & 8 \\
\hline CP $(\%)$ & 18,60 & 14,55 & 12,76 & 8,50 & 3,32 & 2,32 & 4,26 & 1,4 & 1,02 \\
\hline
\end{tabular}


Potentiel d'affaissement (CP \%)

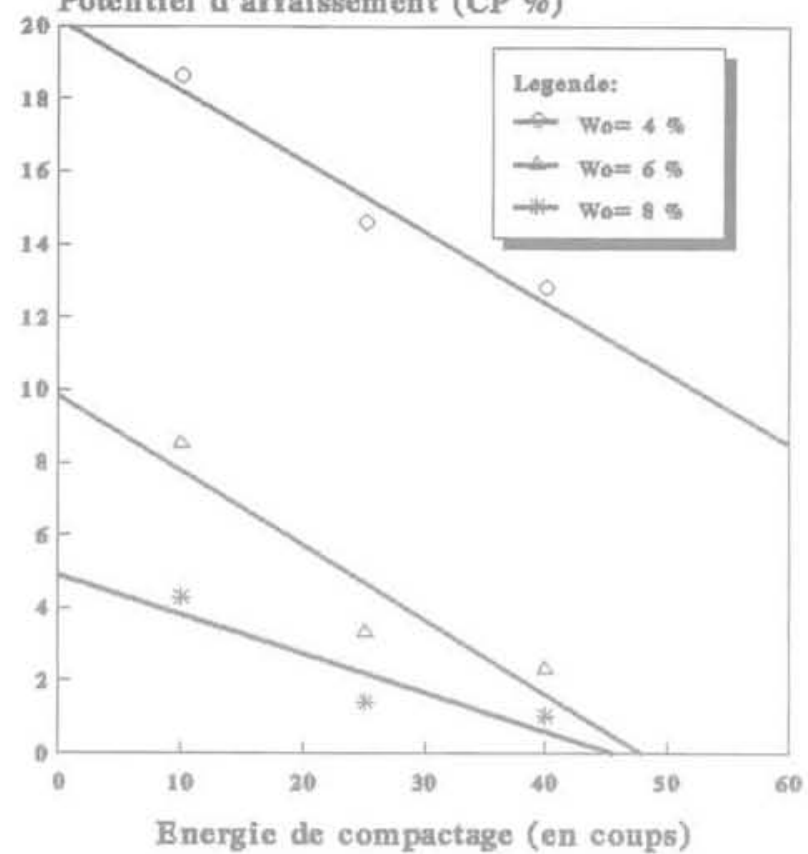

คiG.6 Infiuence de l'énergie de compactage sur $\mathrm{CP}$ (pour $0 \%$ de bitume).

- il y a une tendance manifeste à l'effondrement du sol non traité qui correspond, dans la classification de Jennings et Knight, aux rubriques allant de $\alpha$ troubles modérés n à « trouble sévères $n$ (un potentiel CP allant de 1,02 à 18,6\%); - le potentiel d'affaissement CP décroit quasi linéairement lorsqu'on augmente la teneur en eau ou l'énergie de compactage.

\section{2}

\section{Sol traité}

Le potentiel d'affaissement varie avec l'un des deux paramètres suivants

- le taux de bitume:

- l'énergie de compactage.

Pour évaluer l'influence de chacun de ces paramétres et pour connaitre les conditions nécessaires pour réduire le potentiel d'affaissement à un degré acceptable, on a analysé l'influence de chaque paramètre séparément.

\section{Influence de l'énergie de compactage}

A l'exception d'un seul cas, $12 \%$ de bitume et 80 coups, aucune autre condition n'a donné un potentiel d'affaissement au-dessous de la valeur limite acceptable (c'est-à-dire $C P<1 \%$ ). Mais il est nécessaire de noter que le potentiel d'affaissement est inversement proportionnel à l'énergie de compactage (Fig. 7). Ceci implique qu'on
Potentiel d'af faissement (CP en \%)

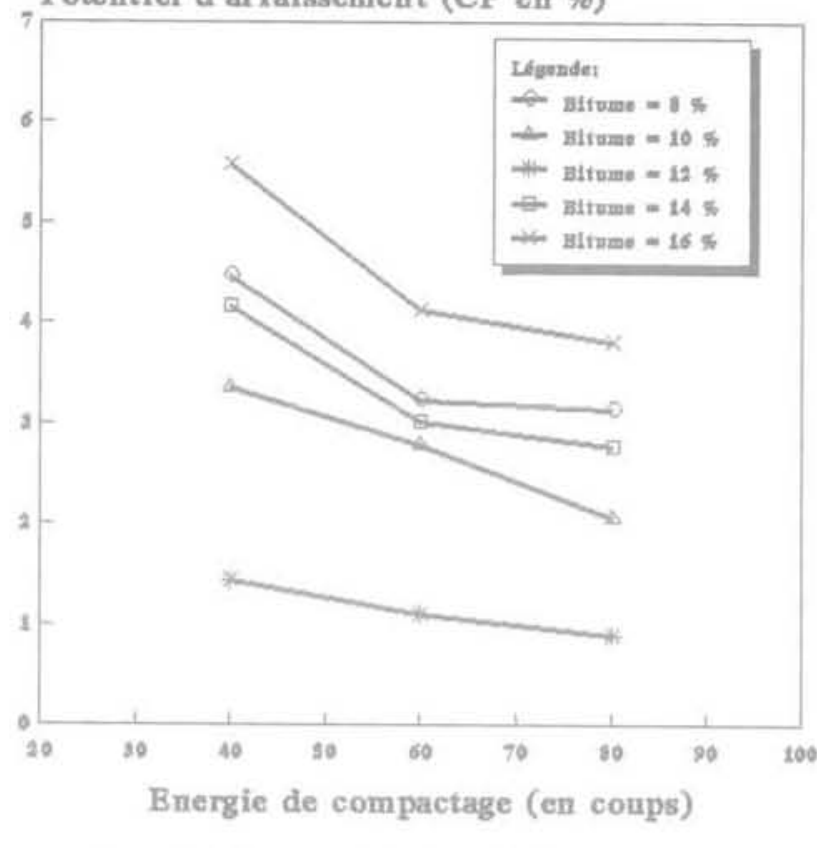

AG.7 Influence de l'énergie de compactage sur $\mathrm{CP}$ du sol traité (pour $W_{0}=4 \%$ ).

ne peut obtenir des valeurs de CP inférieures à 1 si on n'augmente pas le nombre de coups à plus de 80 . Cela représente l'un des inconvénients majeurs de ce type de traitement. En pratique, il faudra donc avoir recours à des engins de compactage assurant une énergie élevée.

\section{Influence de la teneur en bitume}

D'après le tableau $V$ et la figure 8 , on remarque qưi $y$ a deux cas :

$1^{\text {er }}$ cas : pour une teneur en bitume $\mathrm{Tb}<12 \%$, le potentiel d'affaissement diminue avec l'augmentation de la teneur en bitume:

$2^{\circ}$ cas : pour une teneur en bitume Tb $>12 \%$, le potentiel d'affaissement augmente avec l'augmentation de la teneur en bitume.

Pour confirmer ces constatations et montrer clairement l'existence de ce seuil, on a calculé le coefficient d'amélioration (BR) pour les différents degrés de compactage d'après la relation:

$$
B R(\%)=\frac{C P_{0}(\%)-C P_{X}(\%)}{C P_{0}(\%)}
$$

avec : BR : Coefficient d'amélioration :

$\mathrm{CP}_{0}$ : Potentiel d'affaissement avant traitement

$\mathrm{CP}_{\mathrm{x}}$ : Potentiel d'affaissement après traitement avec $\mathrm{x}$ $(\%)$ de bitume.

TABLEAUV Valeurs de CP pour sol traité ( $w=4 \%$ ).

\begin{tabular}{|c|c|c|c|c|c|c|c|c|c|c|c|c|c|c|c|}
\hline \multirow{2}{*}{$\begin{array}{l}\text { Teneur en bitume }(\%) \\
\text { Nombres de coups } \\
\end{array}$} & \multicolumn{3}{|c|}{8} & \multicolumn{3}{|c|}{10} & \multicolumn{3}{|c|}{12} & \multicolumn{3}{|c|}{14} & \multicolumn{3}{|c|}{16} \\
\hline & 40 & 60 & 80 & 40 & 60 & 80 & 40 & 60 & 80 & 40 & 60 & 80 & 40 & 60 & 80 \\
\hline$C P(\%)$ & 4,47 & 3,23 & 3,13 & 3,35 & 2,79 & 2,05 & 1.43 & 1.10 & 0,89 & 4,16 & 3,02 & 2,77 & 5,57 & 4,12 & 3,80 \\
\hline
\end{tabular}




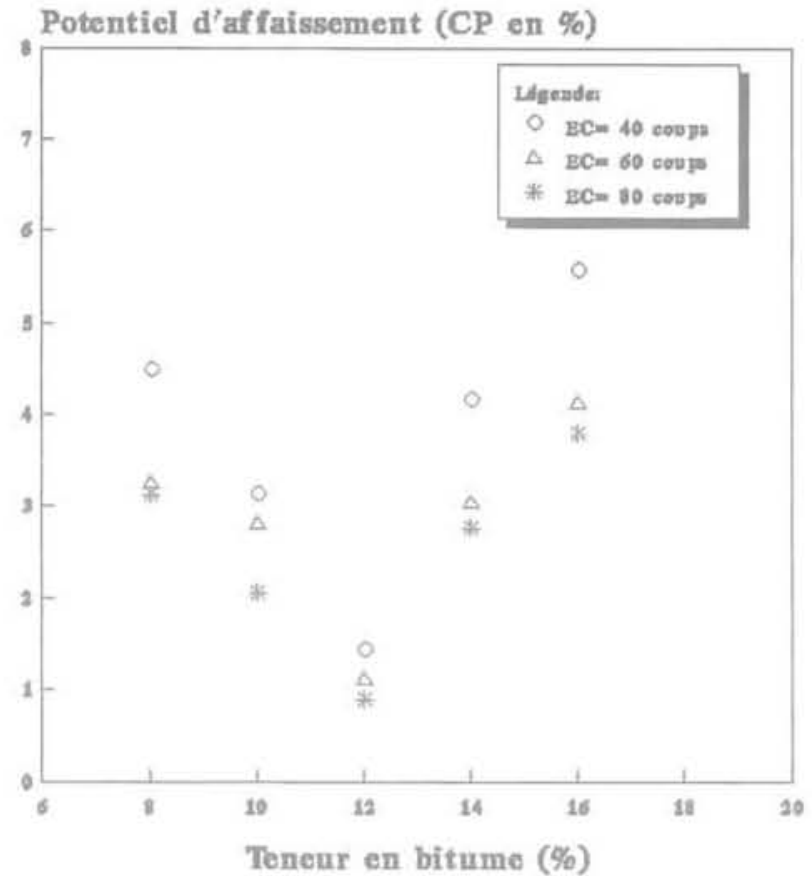

FG. I Influence de la teneur en bitume sur CP (pour Wo $=4 \%$ ).

Les résultats du coefficient d'amélioration, pour une énergie de compactage de 40 coups, sont donnés dans le tableau VI et représentés sur la figure 9. Ceux-ci montrent que la variation du coefficient BR avec le taux du bitume a la forme de la courbe Proctor où le BR atteint une valeur maximale dans l'intervalle [10\%$12 \% 1$ de bitume. Les mêmes observations ont été faites pour les énergies de compactage de 60 et 80 coups. On peut donc conclure que la teneur en bitume donnant le coefficient d'amélioration optimal est située dans l'intervalle [10\%-12 \%]. Mais il est indispensable de noter que cette valeur optimale du BR ne correspond toujours pas à un potentiel d'affaissement CP inférieur à 1, surtout pour les énergies de compactage inférieures à 80 coups.

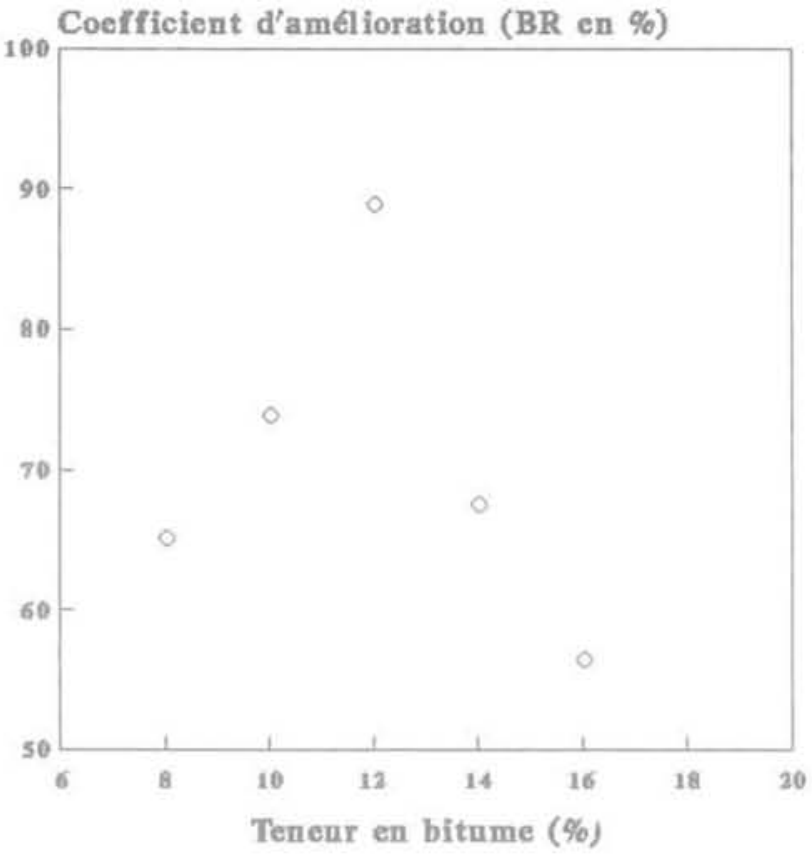

FIG. 9 Variation du coefficient d'amélioration (BR) avec la teneur en bitume (pour EC = 40 coups).

\subsection{3}

\section{Influence de la plasticité du bitume sur l'effondrement}

Pour étudier cette influence, on a calculé le degré d'affaissement DC introduit, pour la première fois, par Ayadat et Belouahri [8] et définit comme :

$$
D C(\%)=\frac{C P t}{C P_{f}} \times 100
$$

avec : DC : Degré d'affaissement ; $\mathrm{CP}_{1}$ : Potentiel d'affaissement à l'instant $(\mathrm{t})$; $\mathrm{CP}_{\mathrm{f}}$ : Potentiel d'affaissement final.

TABtEAu n Valeurs du coefficient d'amélioration (BR).

\begin{tabular}{l|c|c|c|c|c}
\hline Feneur en bitume & $8 \%$ & $10 \%$ & $12 \%$ & $14 \%$ & $16 \%$ \\
\hline Energie de compactage & 40 & 40 & 40 & 40 & 40 \\
\hline $\mathrm{CP}_{0}(\%)$ & 12,76 & 12,76 & 12,76 & 12,76 & 12,76 \\
\hline $\mathrm{CP},(\%)$ & 4,47 & 3,35 & 1,43 & 4,16 & 5,57 \\
\hline $\mathrm{BR}(\%)$ & 65 & 73,75 & 88,80 & 67,40 & 56,38 \\
\hline
\end{tabular}

TABLEAU VIII Valeurs du degré d'affaissement (DC) $\left(W=4 \%, E_{C}=40\right.$ coups et $\left.\mathrm{Tb}=12 \%\right)$.

\begin{tabular}{l|c|c|c|c|c}
\hline Temps (mn) & 1 & 10 & 30 & 50 & 60 \\
\hline $\mathrm{CP}(\%)$ Sol non traité & 10,62 & 12,26 & 12,58 & 12,67 & 12,74 \\
\hline $\mathrm{CP},(\%)$ Sol non traité & 12,76 & 12,76 & 12,76 & 12,76 & 12,76 \\
\hline $\mathrm{DC}(\%)$ Sol non traité & 83,25 & 96,08 & 98,61 & 99,33 & 99,87 \\
\hline $\mathrm{CP}(\%)$ Sol traité & 0,0005 & 0,015 & 0,035 & 0,0456 & 0,06 \\
\hline $\mathrm{CP},(\%)$ Sol traité & 1,43 & 1,43 & 1,43 & 1,43 & 1,43 \\
\hline $\mathrm{DC}(\%)$ Sol traité & 0,036 & 1,03 & 2,48 & 3,19 & 4,16 \\
\hline
\end{tabular}


Les résultats obtenus montrent clairement qu'il y a une grande différence entre la variation de DC en fonction du temps pour un sol non traité et pour le même sol traité avec un taux donné de bitume, et qu'on peut résumer comme suit :

- sol non traité : le phénomène d'affaissement se manifeste brusquement. Il atteint $80 \%$ du tassement final dans les deux premières minutes après l'inondation (Tableau VII et Fig. 10). Ce résultat confirme les travaux de Ayadat et Belouahri [8] :

- sol traité : l'affaissement s'effectue lentement, à une vitesse presque uniforme, au cours de l'inondation de l'êchantillon (Fig. 10).

Ce comportement peut être expliqué comme suit: - Les liaisons entre les grains du sol non traité sont assurées par la succion capillaire et en grande partie par les ponts argileux. L'élimination de cette succion ou la réduction de la résistance des liaisons argileuses entre les gros grains, causées par l'addition d'eau à l'échantillon, entraînent un réarrangement radical des grains et même un mouvement des particules fines à travers le sol, d'un horizon ou d'un niveau à un autre (phénomène de suffusion [8]). Ceci implique l'effondrement brusque du sol. La magnitude et la vitesse de l'affaissement dépendent, essentiellement, de l'indice des vides initial et du type des liaisons entre les grains. Le potentiel d'affaissement est d'autant plus élevé que le sol possède un indice des vides plus grand, et le tassement est d'autant plus rapide que le sol contient un pourcentage des fines particules plus élevé [8].

- L'échantillon du sol traité se compose de grains de sol, de grains de sol enrobés par le bitume, de bitume et des vides. L'inondation de l'échantillon provoque la rupture de quelques liaisons intergranulaires non touchées par le traitement. Ceci entraine le tassement de l'échantillon qui sera dans ce cas petit, puisqu'une grande partie des vides est remplie par le bitume. Ce tassement est aussi caractérisé par une vitesse lente et ceci est dû, essentiellement, au fait que le traitement par du bitume entraine une décroissance de la perméabilité du sol et plus celui-ci devient imperméable, plus l'écoulement d'eau à travers les vides devient lent, ce qui réduit considérablement la variation en fonction du temps du tassement du sol, causé par saturation.

L'amplitude du tassement final, dans ce cas, ne dépend pas que du volume des vides libres dans le sol, mais dépend aussi de la compressibilité du bitume libre en enrobant et les grains. Ceci explique, en premier lieu, I'uniformité de la vitesse du tassement dans ce type de traitement et, en deuxième lieu, l'augmentation du potentiel d'affaissement avec l'augmentation du taux de bitume au-delà de la teneur $\mathrm{Tb}=12 \%$. L'existence d'une grande quantité de bitume dans les vides augmente la compressibilité du sol donc augmente la valeur de son tassement final. Après inondation, la compressibilité du sol affaissable traité devient dépendante de celle du bitume.

Sachant que la compressibilité du bitume à un instant quelconque dépend de la durée écoulée depuis l'instant de son émulsion (c'est-à-dire le degré de durcissement du bitume), on peut conclure que ce procédé est lent, et ceci conduit à noter un autre désavantage dans cette méthode de traitement.

Enfin, au-delà d'une teneur en bitume de $16 \%$, le sol traité devient de plus en plus imperméable, et le

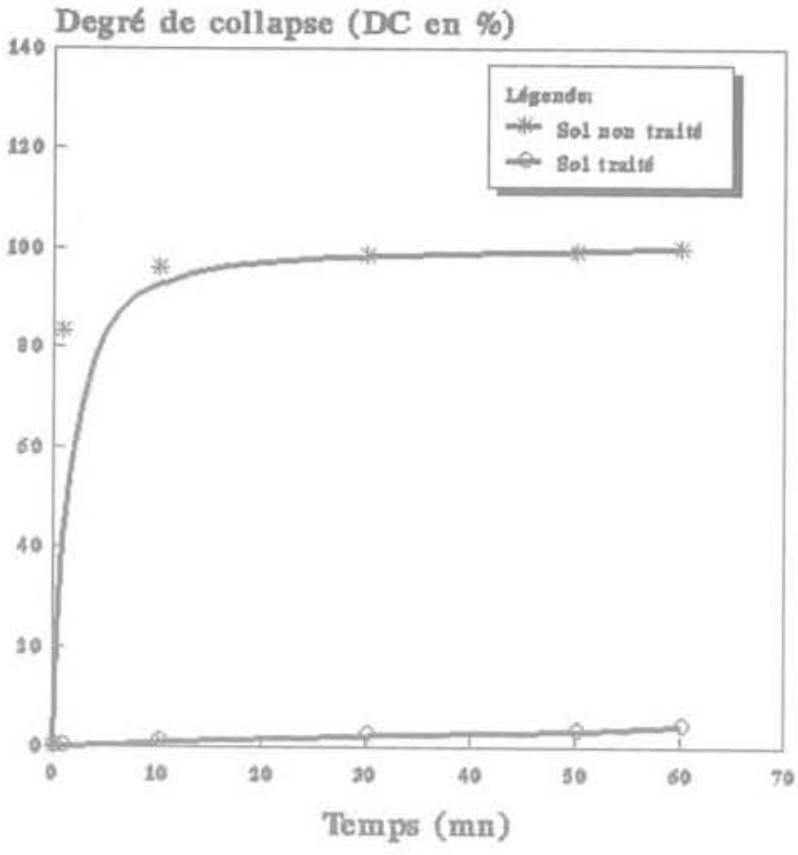

FG.10 Variation du degré de collapse (DC) avec le temps (pour $W_{0}=4 \%, E C=40$ coups et la teneur en bitume $=12 \%$ ).

mouillage de l'échantillon devient de plus en plus difficile, voire impossible. Ceci entraîne une réduction du tassement final, donc du potentiel d'affaissement, lorsque le taux du bitume est supérieur à $16 \%$. Ce résultat n'était pas l'objectif de cette étude, puisque l'on a cherché, évidemment, à mettre le moins possible de bitume pour des raisons économiques.

\section{5}

\section{Conclusion}

L'objet de cette recherche a été de déterminer les conditions optimales du traitement par du bitume d'un sol effondrable, de profondeur inférieure à $4 \mathrm{~m}$, situé en zones arides ou semi-arides.

Les résultats montrent que, pour obtenir un sol non effondrable pour la quantité minimale d'eau retenue (4\%), il faut utiliser un taux de bitume compris entre 10 et $12 \%$ et une énergie de compactage élevée (plus de 80 coups). Nous avons montré aussi qu'il est toujours possible d'obtenir un sol non effondrable en augmentant la teneur en bitume au-delà de $16 \%$, mais ce n'était pas l'objectif de cette recherche.

A l'issue de ces travaux, on doit constater que les résultats obtenus dépendent certainement de la nature des matériaux utilisés pour la reconstitution d'un sol effondrable, du mode de compactage et aussi du temps écoulé entre le traitement du sol et le moment où l'on procède à la saturation de l'échantillon dans le moule de l'cedomètre. Ce temps a une influence sur le degré de durcissement du bitume. Pour cela, nous souhaitons que d'autres études soient effectuées, en utilisant d'autres types de matériaux de reconstitution, d'autres méthodes de compactage, en faisant varier le temps de durcissement du bitume avant saturation de l'échantillon et en confirmant ces résultats sur des échantillons de sols naturels traités. 
Enfin, on notera qu'il est possible d'appliquer cette méthode de traitement s'il n'existe pas d'autres procédés moins coûteux, tels que le traitement à la chaux.
Ceci est dù, essentiellement, au fait que ce type de traitement est lent et nécessite un degré de compactage plus élevé.

\section{$\overline{\text { Bibliographie }}$}

Ayadat T. Cherabli A. - Traitement d'un sol affaissable par du ciment CPA 325. Annales cie l7TBTP, $n^{8} 530$, p. $34-43$ janvier 1995

Ayadat T., Bensalem A. Bankerri A. Traitement d'un sol affaissable par la chaux. Revue française de gejotechnique $n^{2} 77.1996$, p. $69-72$

Merrouche Z, Saidat A. - Traitement d'un sol affaissable par du ciment alumineux fondu. Mémoire de fin d'étude. Université de M'Sila, juin 1995.
Ayadat T. - Collapse of stone column foundations due to inundation. A paraittre dans le Journzel of Technology (Algérie).

Bara J.P. - Collapsing soils. ASCE annua: convention, Philadelphia 1976, reprint 1979

Avadat T. Abbèche K. - Evaluation et nouvelles interprétations des critères de précliction des sols affaissables. A paraitre dans la revue Algérie-Equipement, 1998.
Jennings J.P., Knight K. - A quide to construction on or with materials exhibiting additional settlement due to col. lapse of grain structure. Proc.6th RCSMFE for Africa, P. 99-105, 1975. Ayaclat 'T., Belouahri B. - Influence di coefficient d'uniformité sur le taux et lamplitude de laffaissement. Revue Française de Géotechnique, $n^{*} 76,1996$, p. $25-35$ 